\title{
Active control of ultrafast electron dynamics in plasmonic gaps using an applied bias
}

\author{
Markus Ludwig, ${ }^{1}$ Andrey K. Kazansky $\odot,{ }^{2,3}$ Garikoitz Aguirregabiria, ${ }^{4}$ Dana Codruta Marinica, ${ }^{5}$ Matthias Falk, \\ Alfred Leitenstorfer, ${ }^{1}$ Daniele Brida, ${ }^{1,6}$ Javier Aizpurua, ${ }^{2,4}$ and Andrei G. Borisov ${ }^{5},{ }^{*}$ \\ ${ }^{1}$ Department of Physics and Center for Applied Photonics, University of Konstanz, D-78457 Konstanz, Germany \\ ${ }^{2}$ Donostia International Physics Center DIPC, Paseo Manuel de Lardizabal 4, 20018 Donostia-San Sebastián, Spain \\ ${ }^{3}$ IKERBASQUE, Basque Foundation for Science, 48013 Bilbao, Spain \\ ${ }^{4}$ Material Physics Center CSIC-UPV/EHU, Paseo Manuel de Lardizabal 5, 20018 Donostia-San Sebastián, Spain \\ ${ }^{5}$ Université Paris-Saclay, CNRS, Institut des Sciences Moléculaires d'Orsay, 91405 Orsay, France \\ ${ }^{6}$ Department of Physics and Materials Science, University of Luxembourg, 162a avenue de la Faïencerie, L-1511 Luxembourg, Luxembourg
}

(Received 18 February 2020; accepted 8 June 2020; published 24 June 2020)

\begin{abstract}
In this joint experimental and theoretical study we demonstrate coherent control of the optical field emission and electron transport in plasmonic gaps subjected to intense single-cycle laser pulses. Our results show that an external $\mathrm{THz}$ field or a minor dc bias, orders of magnitude smaller than the optical bias owing to the laser field, allows one to modulate and direct the electron photocurrents in the gap of a connected nanoantenna operating as an ultrafast nanoscale vacuum diode for lightwave electronics. Using time-dependent density functional theory calculations we elucidate the main physical mechanisms behind the observed effects and show that an applied dc field significantly modifies the optical field emission and quiver motion of photoemitted electrons within the gap. The quantum many-body theory reproduces the measured net electron transport in the experimental device, which allows us to establish a paradigm for controlling nanocircuits at petahertz frequencies.
\end{abstract}

DOI: 10.1103/PhysRevB.101.241412

The interaction of intense short laser pulses with matter provides access to the dynamics of electronic excitations in a highly nonlinear regime characterized by emission of energetic electron bursts of subcycle duration and by generation of high harmonics used to track the evolution of the quantum systems at attosecond time scales [1-4]. For metal surfaces and metal nanoparticles, the coupling of light with collective electronic excitations (plasmons) allows one to engineer enhanced optical fields at the hot spots with characteristic sizes well below the diffraction limit $[5,6]$. Thus the optical field emission regime can be reached for incident field strengths significantly smaller than those required for molecular and atomic species in the gas phase $[4,7,8]$. In contrast to electron photoemission via multiphoton absorption, optical field emission can be addressed as an electron tunneling at the metal/vacuum interface in a situation where the potential barrier is strongly modified by the instantaneous optical field.

The in-depth studies performed for metallic surfaces, metallic nanotips, and plasmonic nanoparticles revealed that multiphoton and optical field electron emission can be manipulated at femtosecond time scales via the carrier-envelope phase (CEP) of the driving laser pulse [9-13]. Using a THz field or a dc applied bias along with the optical excitation offers additional possibilities [14-19] for the coherent control of electron dynamics. In this context, among the plasmonic nanoobjects that can be applied for light wave electronics [20-25], the dimer antenna with a nanoscale gap is particularly relevant. On the one hand, the coupling between

*andrei.borissov@u-psud.fr electrons and photons in narrow gaps of dimer antennas leads to light emission originating from inelastic electron tunneling events [26-29]. On the other hand, the highly nonlinear optical field electron emission process [21,24,25,30,31] as well as optically assisted electron tunneling [32-34] allow for rectification at optical frequencies and CEP control of the electron transport across the junction $[23,35,36]$.

In this Rapid Communication, we demonstrate coherent control of the net electric current in a nanocircuit comprising a single bowtie nanoantenna with a $6 \mathrm{~nm}$ wide gap as presented in Fig. 1. We show that a dc field two orders of magnitude smaller than the optical field induced in the gap by an incident single-cycle laser pulse allows for controlling and directing the petahertz currents of the optically emitted electrons and thus the electron transport in the device. The control strategy reported here and based on the dc bias applied between the antenna arms operates simultaneously with earlier studied coherent control using the CEP of the incident pulse $[23,35,36]$. Our study thus establishes a conceptual basis to extend the application of static or $\mathrm{THz}$ fields beyond the control of electron (photo)emission from metallic tips [14-19] and electron tunneling [37-39]. Along with dielectric, semiconductor [40-43], graphene-based [20,44], and tunneling [36] structures, the theoretical and experimental realization demonstrated here, analog to an ultrafast rectifying vacuum diode (see also [31]), paves the way towards petahertz electronics [45].

The gold bowtie nanoantenna has been fabricated by electron beam lithography on a silica substrate. The two arms of the antenna are interfaced macroscopically [see Fig. 1(b)] with a transimpedance amplifier which allows for a readout of the optically driven currents via a lock-in scheme. An 


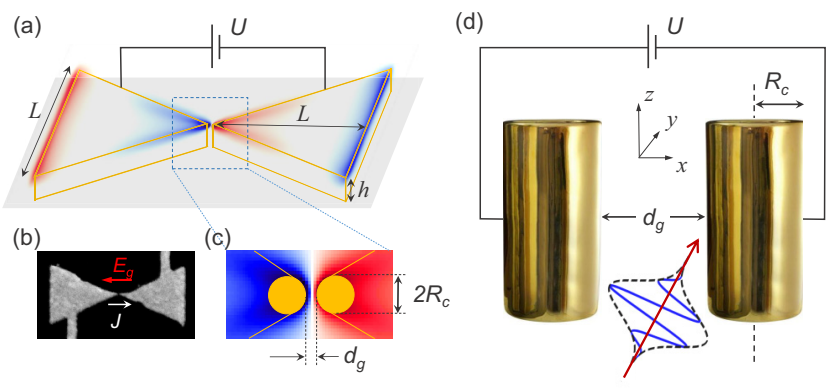

FIG. 1. Conceptual sketch of the system. (a) Induced charge density (red, positive; blue, negative) associated with the bonding dipolar plasmon mode of the bowtie nanoantenna embedded in a macroscopic circuit (height $h=20 \mathrm{~nm}$ and lateral size $L=360 \mathrm{~nm}$ ). Results are obtained from the solution of the classical Maxwell equations for the mode frequency $\omega_{0}=0.68 \mathrm{eV}$. (b) Scanning electron micrography (SEM) of the actual experimental device. The red, $E_{g}$, and white, $J$, arrows show the direction of the instantaneous electric field and electron current in the gap, respectively. (c) Zoom of panel (a) into the gap region. The size of the gap $d_{g}=6 \mathrm{~nm}$ and the curvature radii of metal tips $R_{c}=5 \mathrm{~nm}$ are obtained from analysis of the SEM images. (d) Theoretical model: system of parallel metallic cylindrical nanowires in vacuum $\left(R_{c}=5 \mathrm{~nm} ; d_{g}=6 \mathrm{~nm}\right)$. The incident single-cycle laser pulse propagates along the $y$ axis and it is linearly polarized with the electric field along the $x$ axis. $(x=0, y=0)$ is set at the center of the gap. The bias $U$ is applied to the left cylinder while the right cylinder is grounded.

additional dc bias can be applied across the antenna gap by means of a bias tee. The ultrafast currents are driven by the electric field transients of single-cycle light pulses of a carrier wavelength of $1250 \mathrm{~nm}$ and a duration of $4.2 \mathrm{fs}$. These pulses are generated by a homemade laser system based on Er:fibers operating at a repetition rate of $80 \mathrm{MHz}$. The passively locked carrier-envelope phase can be controlled with subcycle precision and a root-mean-square stability of $10 \mathrm{mrad}$ [35].

Because of the strong enhancement of the field of the incident laser pulse, the optical field emission and electron transfer processes are dominated by the antenna gap. The main physics behind the experimental observations can thus be captured theoretically using the model system which reproduces the gap geometry of the actual device [35]. In practice we consider two parallel gold cylinders in vacuum, as sketched in Fig. 1(d). The gold nanoparticles are represented using the free electron, jellium metal (JM) approximation [46]. Together with the simplified geometry this allows one to perform the time dependent density functional theory [47-50] (TDDFT) calculations of the photoemission and transport dominated by the dynamics of valence electrons [7-9,35] and account for the plasmon effects [51-53]. We use the real-time propagation of the Kohn-Sham orbitals [47] based on the pseudospectral approach [54-57].

Prior to the discussion of the actual experimental data, we start by analyzing the results of the TDDFT calculations for a model system: the nanowire dimer subjected to an idealized Gaussian pulse. Thus the main physical concepts and effects can be demonstrated in a clear way, which facilitates the understanding of the results for an actual device.

The electric field $E_{\mathrm{IR}}(t)$ of the incident $x$-polarized Gaussian pulse with carrier frequency $\omega$, duration $\tau=2 \pi / \omega$, and $\operatorname{CEP} \phi$ is given by $E_{\mathrm{IR}}(t)=E_{0} e^{-t^{2} / \tau^{2}} \cos (\omega t+\phi)$. The amplitude is $E_{0}=9 \mathrm{~V} / \mathrm{nm}$, and $\omega$ is set well below the plasmon resonance of the model nanowire dimer $\omega_{\mathrm{DP}}=5.9 \mathrm{eV}$ [35].

Thus the self-consistent field in the middle of the gap, $E_{g}(t)$, calculated with TDDFT features the same temporal profile as the incident pulse, $E_{g}(t) \simeq 1.7 \times E_{\mathrm{IR}}(t)$. The moderate $(\times 1.7)$ field enhancement in this case results in a maximum ac electric field value reached in the gap, $E_{\mathrm{gm}} \approx 15 \mathrm{~V} / \mathrm{nm}$. The associated Keldysh parameter $\gamma=\omega \sqrt{2 m_{e} \Phi} / e E_{\mathrm{gm}} \lesssim 1$ indicates that the system is predominantly in the optical field emission regime [9,58-65]. Here, $m_{e}$ is the electron mass, $e$ stands for elementary charge, and $\Phi=5.5 \mathrm{eV}$ is the work function of gold [66].

The effect of an applied bias $U$ on the electron transfer across the gap is shown in Fig. 2. For $U=0$ we obtain the characteristic sinusoidal variation of the net electron current as a function of the CEP of the incident pulse [23,35,67]. Here the net electron current is defined as the number of electrons transferred per pulse between the nanowires; its sign is defined with respect to the positive direction of the $x$ axis. If a relatively small bias $U=2 \mathrm{eV}$ is applied across the gap, the entire CEP dependence of the electron transfer shifts towards negative values. The current offset is larger for higher frequency of the pulse, and, for $\omega=1.2 \mathrm{eV}$, the positive electron transfer is nearly blocked. This is a remarkable result since the applied dc bias is more than one order of magnitude smaller than the optical bias, $U_{\text {opt }}=90 \mathrm{~V}$, reached for the $E_{\mathrm{gm}}=15 \mathrm{~V} / \mathrm{nm}$ ac field in the $d_{g}=6 \mathrm{~nm}$ gap.

Since for the present size of the gap the tunneling is negligible, no dc current flows through the junction without the optical field. The calculated modification of the electron transport by an applied bias $U$ originates then from its effect on the ejection and propagation of the photoemitted electrons. In order to elucidate the main mechanisms behind the effect of an applied bias, we analyze in Fig. 3 the dynamics of electron currents in the gap of the model cylinder dimer. The spatiotemporal profiles of the electron density current are shown for the $\mathrm{CEP}=0.875 \pi$ corresponding to the maximum positive electron transfer. To render the effects clearer we compare the results obtained with no applied bias and those obtained with a relatively high bias of $U=8 \mathrm{eV}$.

As a common feature, subsequent half periods with positive (negative) field in the gap, $E_{g}(t)$, lead to the ejection of electrons from the surface of the right (left) cylinder. The electron burst of highest intensity is emitted during the central half period of the pulse around $t=0$. Fast electrons cross the gap on the subcycle time scale; however, there are many electrons that do not cross the gap within the half period of the optical field. When $E_{g}(t)$ changes sign their trajectories are reversed towards the parent cylinder. Some of the electrons are trapped at quiver trajectories inside the gap [67]. It appears that these electrons have a low drift velocity which can be strongly affected by even a small applied bias. This result is central for the active control strategy proposed here. In Fig. 3(c) the slow electrons directed by a dc field can be seen as a negative electron current at large time delays. 

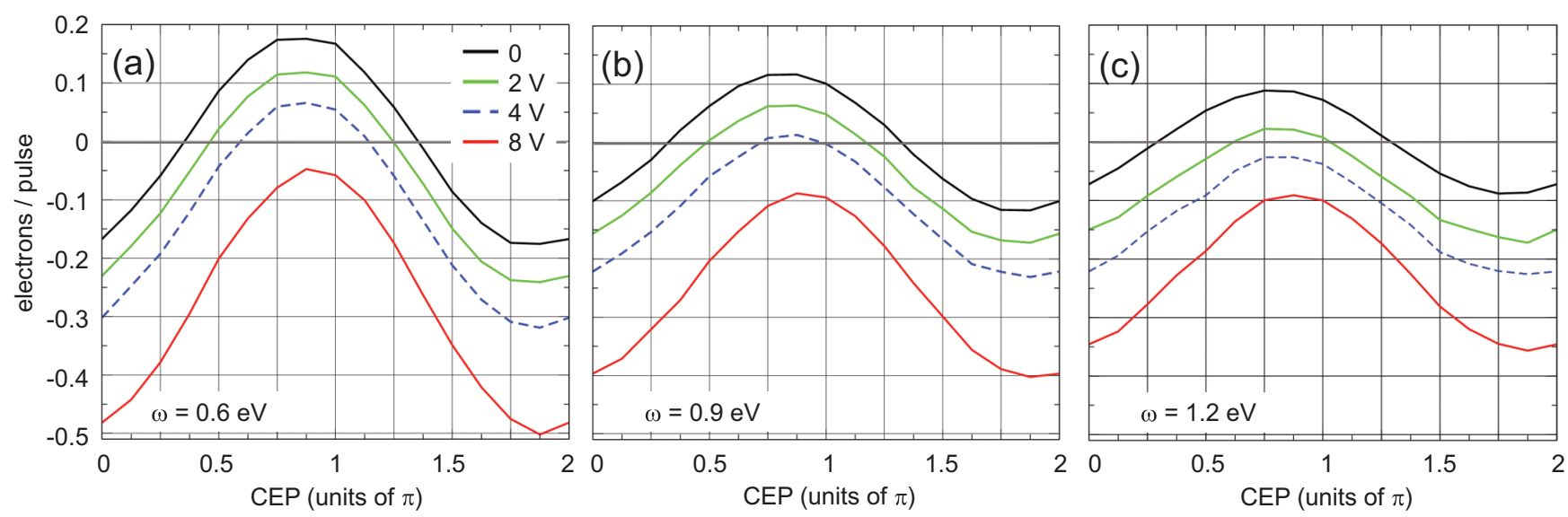

FIG. 2. Calculated with TDDFT dc bias control of the strong field emission and electron transport in the gap of the nanowire dimer subjected to a single-cycle optical pulse with carrier frequency (a) $\omega=0.6 \mathrm{eV}$, (b) $\omega=0.9 \mathrm{eV}$, and (c) $\omega=1.2 \mathrm{eV}$. The number of electrons transferred between the cylinders per optical pulse and per unit height is shown as a function of the CEP for different applied bias $U$ as explained in the inset.

Similar results are obtained with different CEP of the incident laser pulse which explains the negative offset of the electron transport.

The distance that an electron emitted at $t=0$ might travel before turning back towards its parent cylinder can be estimated as $2 X_{q}$. Here $X_{q}=e E_{\mathrm{gm}} / m_{e} \omega^{2}$ is the quiver amplitude of an electron subjected to an optical field of amplitude $E_{g m}$ and frequency $\omega$. With increasing $\omega$, the quiver amplitude decreases: $2 X_{q}=5.6 \mathrm{~nm}(\omega=0.6 \mathrm{eV}) ; 2 X_{q}=2.5 \mathrm{~nm}(\omega=$ $0.9 \mathrm{eV}), 2 X_{q}=1.4 \mathrm{~nm}(\omega=1.2 \mathrm{eV})$, and more electrons experience quiver motion [35,67]. This in turn explains a stronger effect of an applied bias with increasing $\omega$ in Fig. 2. It is worth noting that, for the dimer antenna, the electromagnetic field is nearly homogeneous inside the gap so that the quiver motion is not quenched in contrast with individual nano-objects [8,68-70].

Along with electron trajectories, the applied bias also modifies the probability of optical field electron emission from the metal surfaces across the gap. This effect is clearly seen when comparing Fig. 3(b) and Fig. 3(c). The intensity of the ejected

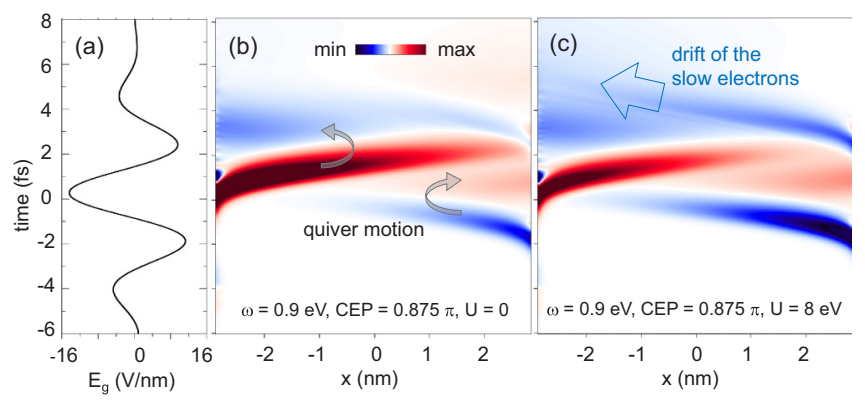

FIG. 3. Electron transport dynamics in the gap for the incident pulse with carrier frequency $\omega=0.9 \mathrm{eV}$ and $\mathrm{CEP}=0.875 \pi$. Panel (a): time dependence of the optical field in the gap $E_{g}(t)$. Panels (b) and (c): spatiotemporal profile of the electron density current $J$ along the cylinder dimer $x$ axis. The TDDFT results are shown as a function of the $x$ coordinate and time for an applied dc bias $U=0$ (b) and $U=8 \mathrm{eV}$ (c). Red (blue) color is used for positive (negative) values of $J$. For further details, see insets. electron bursts increases (decreases) when the optical and dc fields are directed in the same (opposite) directions. However, for the large optical fields and small applied bias relevant in our experimental conditions, the trajectory effect dominates as we further discuss in the Supplemental Material [71].

The theoretical findings in the simple model system demonstrate that, in addition to the CEP control of the electron transport and electron emission reported previously, a weak bias applied across the nanogap can be used for coherent control of the electron currents associated with optical field emission induced by single-cycle laser pulses. This prediction is fully confirmed in our experiments for a nanocircuit comprising the bowtie plasmonic antenna as summarized in Fig. 4.

The field driving the electron emission and transport in the gap of the bowtie antenna is determined by the resonant plasmonic response to the incident laser pulse. The total field in the gap is then characterized by (i) a strong enhancement, (ii) phase shift of the induced field, and (iii) extended time duration as compared to the incident pulse, as shown in Fig. 4(a) (compare red and blue dashed lines). In the TDDFT calculations we limit the field evolution to the central and most intense part of the pulse (black curve) dominating the optical field emission. The incident pulse is set such that the self-consistent field in the gap of the model cylinder dimer reproduces the classical result from Maxwell's equations for the actual device.

In Fig. 4(b) we show the measured and calculated net electron current through the junction as a function of freespace amplitude of the transient $E_{0}$ (experiment) and of the maximum field reached in the gap, $E_{\mathrm{gm}}$ (theory). Along with TDDFT calculations we also show the results obtained using the simple man's model (SMM) [7,8,72-75] based on Fowler-Nordheim tunneling through the metal/vacuum interface [76,77] followed by the classical electron motion within the gap as detailed in the Supplemental Material. To compare with the experimental data, the electron transfer per unit length of the nanowire as calculated with TDDFT is multiplied by the height of the bowtie $(h=20 \mathrm{~nm})$. Results are shown for the CEP corresponding to the maximum of the net electron current without bias [see panels (c), (d), and (e)]. 

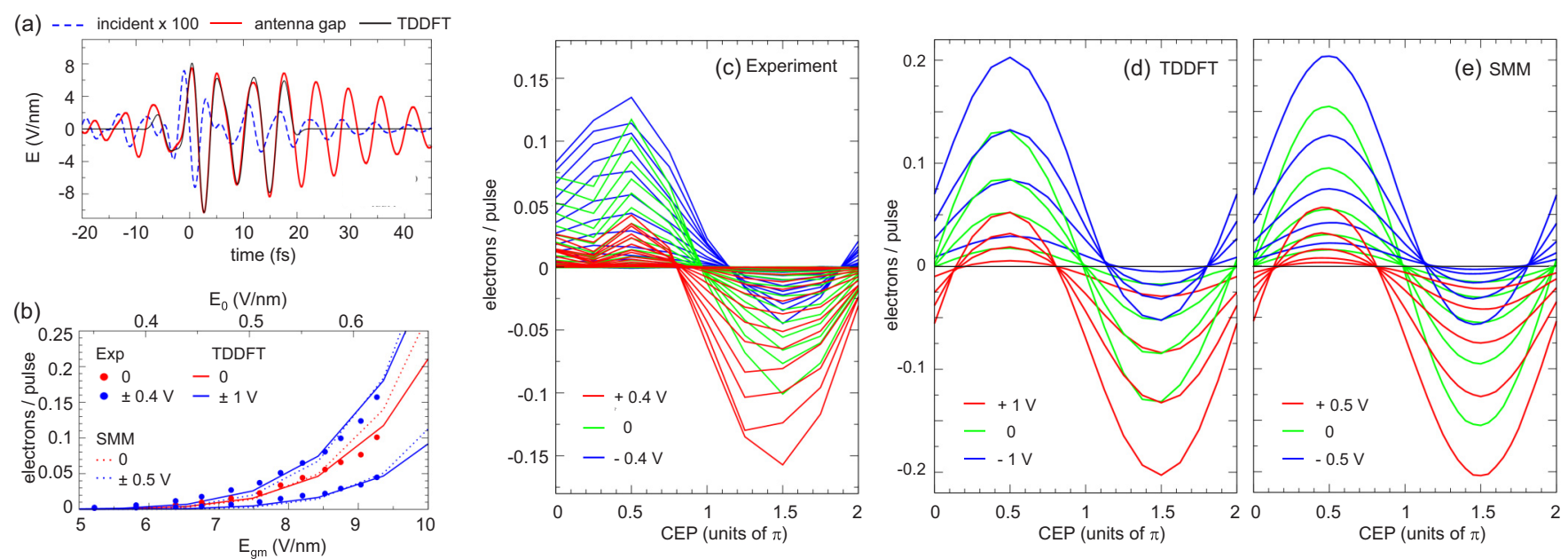

FIG. 4. Coherent control of the electron transport in the gap of the connected bowtie nanoantenna. Panel (a): time dependent electric field. Free-space experimental transient with $\mathrm{CEP}=0.49 \pi$ (dashed blue line, scaled by $\times 100) ; E_{g}(t)$ calculated with classical Maxwell's equations (TDDFT) in the middle of the gap is shown with red (black) line. Panel (b): net electron current (number of electrons transferred per pulse) through the gap of the bowtie for CEP $=0.49 \pi$. Circles: experimental data as a function of the amplitude of the free-space transient $E_{0}$; solid (dashed) line TDDFT (SMM) results calculated as a function of the maximum field reached in the gap $E_{\mathrm{gm}}$. The color code indicates the applied bias as explained in the inset. Panels (c),(d),(e): the CEP dependence of the net electron current. Sets of lines of the same color correspond to the results obtained with a fixed applied bias $U$ and varying the intensity of the laser. In experiment (c) $E_{0}$ is varied between 0.1 and $0.62 \mathrm{~V} / \mathrm{nm}$ in steps of $0.04 \mathrm{~V} / \mathrm{nm}$. The theoretical TDDFT (d) and SMM (e) data correspond to $E_{\mathrm{gm}}=8.32,8.84,9.36,9.88,10.4 \mathrm{~V} / \mathrm{nm}$.

The calibration of the optical field amplitude in the gap $E_{\mathrm{gm}}$ used in the TDDFT and SMM has been performed from the comparison between theory and experiment for the $U=0$ case. This calibration has been then used throughout this study [78].

The qualitative agreement between the experiments, quantum TDDFT, and classical SMM calculations in Fig. 4(b) is remarkable. It follows from our experiments that the $\pm 0.4 \mathrm{eV}$ variation of the applied bias allows one to change the net electron current in the device by nearly a factor of two as compared to the $U=0$ case. We emphasize that this small bias is controlling the optical field emission current since without the laser pulse the tunneling current is negligible for this size of the gap. It is also worth noting that the optical bias in the $6 \mathrm{~nm}$ gap reaches $60 \mathrm{eV}$, i.e., it is 150 times larger than the dc bias. Noteworthy, the effect of the applied bias shown in Fig. 4 is significantly larger than the one calculated for the model system in Fig. 2. While the electron excursion $2 X_{q}=1.7 \mathrm{~nm}$ [79] is within the range of the values explored for the model system, in the actual experimental situation the field in the gap lasts much longer because of the plasmonic ringing. As we show using an intuitive picture of classical trajectories in the Supplemental Material, this plasmonic ringing significantly increases the probability that the emitted electron will be trapped in a quiver trajectory strongly sensitive to the applied bias. In fact, the truncated time evolution of the electric field as imposed by the computational constraints in the TDDFT results in a larger applied bias $U= \pm 1 \mathrm{eV}$ needed to reproduce experimental data. This problem is avoided in the classical SMM.

Finally, in Figs. 4(c), 4(d) and 4(e), we demonstrate coherent control of the electron current in the nanocircuit using both the CEP of the transient and the applied bias. The CEP dependence of the net electron current is measured and calculated for positive and negative values of the applied bias while varying the intensity of the incident pulse. Because of the extremely strong nonlinearity of the optical field emission process the number of the emitted electrons rapidly varies with the intensity of the laser pulse leading to the corresponding scaling of the results. Since only the relative CEP can be controlled experimentally, the absolute value of the CEP in Fig. 4(c) is then determined from the TDDFT results. Once the CEP is set, theory reproduces the experimental observations. Our experimental and theoretical results demonstrate that a small dc bias produces an offset of the entire CEP dependence of the net electron current. In the situation considered in our work, the electron transport can be nearly blocked in a given direction.

In conclusion, in this combined experimental and theoretical study we demonstrated coherent active control of the optical field emission and associated electron current in a nanocircuit comprising a plasmonic gap in a connected bowtie antenna. The active control strategy proposed here is based on the simultaneous exploitation of the CEP of an incident singlecycle optical pulse and of an additional dc bias applied across the gap. We identify the quiver motion of the photoemitted electrons and the strong nonlinearity of the optical field emission as principle mechanisms that allow one to control the electron transport across the gap with a weak dc field and in the absence of any significant background tunneling current. While being more than an order of magnitude smaller than the optical field, the dc field owing to the applied bias might completely block the electron transport so that the system operates as a rectifying controllable petahertz diode. Similarly, one can reach a situation where a specific CEP drives a maximum current, while reversing the bias results in no current at all. This generates an analog of an I or O logic device as it is currently used in CMOS technology. While 
demonstrated with an applied dc bias, the control strategy proposed in this Rapid Communication also applies to the case of a plasmonic gap illuminated with single-cycle optical and $\mathrm{THz}$ pulses and thus bears a promise for applications in lightwave electronics.

The support from the Department of Education of the Basque Government through Project No. PI2017-30 (J.A., A.K.K., and G.A.) and Project No. IT1164-19 for con- solidated groups (J.A.) is gratefully acknowledged. A.K.K. acknowledges support from Project No. FIS2016-76617$\mathrm{P}$ of the Spanish Ministry of Economy and Competitiveness. D.B. and A.L. acknowledge support of the Deutsche Forschungsgemeinschaft through the Emmy Noether programme (No. BR 5030/1-1) and the collaborative research center SFB 767. D.B. acknowledges the support by the European Research Council through Grant No. 819871 (UpTEMPO).
[1] M. Protopapas, C. H. Keitel, and P. L. Knight, Rep. Prog. Phys. 60, 389 (1997).

[2] P. B. Corkum and F. Krausz, Nat. Phys. 3, 381 (2007).

[3] F. Krausz and M. Ivanov, Rev. Mod. Phys. 81, 163 (2009).

[4] M. F. Ciappina, J. A. Pérez-Hernández, A. S. Landsman, W. A. Okell, S. Zherebtsov, B. Förg, J. Schötz, L. Seiffert, T. Fennel, T. Shaaran, T. Zimmermann, A. Chacón, R. Guichard, A. Zaïr, J. W. G. Tisch, J. P. Marangos, T. Witting, A. Braun, S. A. Maier, L. Roso, M. Krüger, P. Hommelhoff, M. F. Kling, F. Krausz, and M. Lewenstein, Rep. Prog. Phys. 80, 054401 (2017).

[5] M. I. Stockman, Opt. Express 19, 22029 (2011).

[6] J. A. Schuller, E. S. Barnard, W. Cai, Y. C. Jun, J. S. White, and M. L. Brongersma, Nat. Mater. 9, 193 (2010).

[7] M. Krüger, M. Schenk, P. Hommelhoff, G. Wachter, C. Lemell, and J. Burgdörfer, New J. Phys. 14, 085019 (2012).

[8] M. Krüger, C. Lemell, G. Wachter, J. Burgdörfer, and P. Hommelhoff, J. Phys. B: At., Mol., Opt. Phys. 51, 172001 (2018)

[9] C. Lemell, X.-M. Tong, F. Krausz, and J. Burgdörfer, Phys. Rev. Lett. 90, 076403 (2003).

[10] P. Dombi, A. Apolonski, C. Lemell, G. G. Paulus, M. Kakehata, R. Holzwarth, T. Udem, K. Torizuka, J. Burgdörfer, T. W. Hänsch, and F. Krausz, New J. Phys. 6, 39 (2004).

[11] A. Apolonski, P. Dombi, G. G. Paulus, M. Kakehata, R. Holzwarth, T. Udem, C. Lemell, K. Torizuka, J. Burgdörfer, T. W. Hänsch, and F. Krausz, Phys. Rev. Lett. 92, 073902 (2004).

[12] M. Krüger, M. Schenk, and P. Hommelhoff, Nature (London) 475, 78 (2011).

[13] B. Piglosiewicz, S. Schmidt, D. J. Park, J. Vogelsang, P. Groß, C. Manzoni, P. Farinello, G. Cerullo, and C. Lienau, Nat. Photon. 8, 37 (2014).

[14] L. Wimmer, G. Herink, D. R. Solli, S. V. Yalunin, K. E. Echternkamp, and C. Ropers, Nat. Phys. 10, 432 (2014).

[15] G. Herink, L. Wimmer, and C. Ropers, New J. Phys. 16, 123005 (2014).

[16] S. Li and R. R. Jones, Nat. Commun. 7, 13405 (2016).

[17] M. Förster, T. Paschen, M. Krüger, C. Lemell, G. Wachter, F. Libisch, T. Madlener, J. Burgdörfer, and P. Hommelhoff, Phys. Rev. Lett. 117, 217601 (2016).

[18] B. Förg, J. Schötz, M. Süßmann, F. nd Förster, M. Krüger, B. Ahn, W. A. Okell, K. Wintersperger, S. Zherebtsov, A. Guggenmos, V. Pervak, A. Kessel, S. A. Trushin, A. M. Azzeer, M. I. Stockman, D. Kim, F. Krausz, P. Hommelhoff, and M. F. Kling, Nat. Commun. 7, 11717 (2016).
[19] L. Wimmer, O. Karnbach, G. Herink, and C. Ropers, Phys. Rev. B 95, 165416 (2017).

[20] B. H. Son, H. S. Kim, J.-Y. Park, S. Lee, D. J. Park, and Y. H. Ahn, ACS Photon. 5, 3943 (2018).

[21] F. Schertz, M. Schmelzeisen, M. Kreiter, H.-J. Elmers, and G. Schönhense, Phys. Rev. Lett. 108, 237602 (2012).

[22] P. Rácz, Z. Pápa, I. Márton, J. Budai, P. Wróbel, T. Stefaniuk, C. Prietl, J. R. Krenn, and P. Dombi, Nano Lett. 17, 1181 (2017).

[23] T. Rybka, M. Ludwig, M. F. Schmalz, V. Knittel, D. Brida, and A. Leitenstorfer, Nat. Photon. 10, 667 (2016).

[24] C. Karnetzky, P. Zimmermann, C. Trummer, C. Duque Sierra, M. Wörle, R. Kienberger, and A. Holleitner, Nat. Commun. 9, 2471 (2018).

[25] P. Zimmermann, A. Hötger, N. Fernandez, A. Nolinder, K. Müller, J. J. Finley, and A. W. Holleitner, Nano Lett. 19, 1172 (2019).

[26] W. Cai, A. P. Vasudev, and M. L. Brongersma, Science 333, 1720 (2011).

[27] H. Lian, Y. Gu, J. Ren, F. Zhang, L. Wang, and Q. Gong, Phys. Rev. Lett. 114, 193002 (2015).

[28] J. C. Prangsma, J. Kern, A. G. Knapp, S. Grossmann, M. Emmerling, M. Kamp, and B. Hecht, Nano Lett. 12, 3915 (2012).

[29] J. Kern, R. Kullock, J. Prangsma, M. Emmerling, M. Kamp, and B. Hecht, Nat. Photon. 9, 582 (2015).

[30] L. Chen, Y. Zhang, G. Chen, and I. Franco, Nat. Commun. 9, 2070 (2018).

[31] T. Higuchi, L. Maisenbacher, A. Liehl, P. Dombi, and P. Hommelhoff, Appl. Phys. Lett. 106, 051109 (2015).

[32] D. R. Ward, F. Hüser, F. Pauly, J. C. Cuevas, and D. Natelson, Nat. Nanotechnol. 5, 732 (2010).

[33] R. Arielly, A. Ofarim, G. Noy, and Y. Selzer, Nano Lett. 11, 2968 (2011).

[34] A. Stolz, J. Berthelot, M.-M. Mennemanteuil, G. C. des Francs, L. Markey, V. Meunier, and A. Bouhelier, Nano Lett. 14, 2330 (2014).

[35] M. Ludwig, G. Aguirregabiria, F. Ritzkowsky, T. Rybka, D. C. Marinica, J. Aizpurua, A. G. Borisov, A. Leitenstorfer, and D. Brida, Nat. Phys. 16, 341 (2020).

[36] M. Garg and K. Kern, Science 367, 411 (2020).

[37] Y.-M. Bahk, B. J. Kang, Y. S. Kim, J.-Y. Kim, W. T. Kim, T. Y. Kim, T. Kang, J. Rhie, S. Han, C.-H. Park, F. Rotermund, and D.-S. Kim, Phys. Rev. Lett. 115, 125501 (2015).

[38] T. L. Cocker, D. Peller, P. Yu, J. Repp, and R. Huber, Nature (London) 539, 263 (2016). 
[39] K. Yoshioka, I. Katayama, Y. Arashida, A. Ban, Y. Kawada, K. Konishi, H. Takahashi, and J. Takeda, Nano Lett. 18, 5198 (2018).

[40] T. M. Fortier, P. A. Roos, D. J. Jones, S. T. Cundiff, R. D. R. Bhat, and J. E. Sipe, Phys. Rev. Lett. 92, 147403 (2004).

[41] A. Schiffrin, T. Paasch-Colberg, N. Karpowicz, V. Apalkov, D. Gerster, S. Mühlbrandt, M. Korbman, J. Reichert, M. Schultze, S. Holzner, J. V. Barth, R. Kienberger, R. Ernstorfer, V. S. Yakovlev, M. I. Stockman, and F. Krausz, Nature (London) 493, 70 (2013).

[42] I. Crassee, L. Gallmann, G. Gäumann, M. Matthews, H. Yanagisawa, T. Feurer, M. Hengsberger, U. Keller, J. Osterwalder, H. J. Wörner, and J. P. Wolf, Struct. Dyn. 4, 061505 (2017).

[43] T. Paasch-Colberg, S. Y. Kruchinin, Ö. Sağlam, S. Kapser, S. Cabrini, S. Muehlbrandt, J. Reichert, J. V. Barth, R. Ernstorfer, R. Kienberger, V. S. Yakovlev, N. Karpowicz, and A. Schiffrin, Optica 3, 1358 (2016).

[44] T. Higuchi, C. Heide, K. Ullmann, H. B. Weber, and P. Hommelhoff, Nature (London) 550, 224 (2017).

[45] J. Schoetz, Z. Wang, E. Pisanty, M. Lewenstein, M. F. Kling, and M. F. Ciappina, ACS Photon. 6, 3057 (2019).

[46] J. P. Perdew, H. Q. Tran, and E. D. Smith, Phys. Rev. B 42, 11627 (1990).

[47] W. Kohn and L. J. Sham, Phys. Rev. 140, A1133 (1965).

[48] E. Runge and E. K. U. Gross, Phys. Rev. Lett. 52, 997 (1984).

[49] M. A. Marques and E. Gross, Annu. Rev. Phys. Chem. 55, 427 (2004).

[50] O. Gunnarsson and B. Lundqvist, Phys. Rev. B 13, 4274 (1976).

[51] J. Zuloaga, E. Prodan, and P. Nordlander, Nano Lett. 9, 887 (2009).

[52] W. Zhu, R. Esteban, A. G. Borisov, J. J. Baumberg, P. Nordlander, H. J. Lezec, J. Aizpurua, and K. B. Crozier, Nat. Commun. 7, 11495 (2016).

[53] A. Varas, P. García-González, J. Feist, F. J. García-Vidal, and A. Rubio, Nanophotonics 5, 409 (2016).

[54] M. D. Feit, J. A. Fleck, and A. Steiger, J. Comput. Phys. 47, 412 (1982).

[55] D. Kosloff and R. Kosloff, J. Comput. Phys. 52, 35 (1983).

[56] R. Kosloff, J. Phys. Chem. 92, 2087 (1988).

[57] C. Leforestier, R. H. Bisseling, C. Cerjan, M. D. Feit, R. Friesner, A. Guldberg, A. Hammerich, C. Jolicard, W. Karrlein, H.-D. Meyer, N. Lipkin, O. Roncero, and R. Kosloff, J. Comput. Phys. 94, 59 (1991).

[58] R. Bormann, M. Gulde, A. Weismann, S. V. Yalunin, and C. Ropers, Phys. Rev. Lett. 105, 147601 (2010).

[59] H. Yanagisawa, M. Hengsberger, D. Leuenberger, M. Klöckner, C. Hafner, T. Greber, and J. Osterwalder, Phys. Rev. Lett. 107, 087601 (2011).

[60] R. G. Hobbs, Y. Yang, A. Fallahi, P. D. Keathley, E. De Leo, F. X. Kärtner, W. S. Graves, and K. K. Berggren, ACS Nano 8, 11474 (2014).
[61] M. E. Swanwick, P. D. Keathley, A. Fallahi, P. R. Krogen, G. Laurent, J. Moses, F. X. Kärtner, and L. F. Velásquez-García, Nano Lett. 14, 5035 (2014).

[62] H. Yanagisawa, S. Schnepp, C. Hafner, M. Hengsberger, D. E. Kim, M. F. Kling, A. Landsman, L. Gallmann, and J. Osterwalder, Sci. Rep. 6, 35877 (2016).

[63] W. P. Putnam, R. G. Hobbs, P. D. Keathley, K. K. Berggren, and F. X. Kärtner, Nat. Phys. 13, 335 (2017).

[64] M. Lehr, B. Foerster, M. Schmitt, K. Krüger, C. Sönnichsen, G. Schönhense, and H.-J. Elmers, Nano Lett. 17, 6606 (2017).

[65] S. V. Yalunin, M. Gulde, and C. Ropers, Phys. Rev. B 84, 195426 (2011).

[66] E. V. Chulkov, V. M. Silkin, and P. M. Echenique, Surf. Sci. 437, 330 (1999).

[67] G. Aguirregabiria, D.-C. Marinica, M. Ludwig, D. Brida, A. Leitenstorfer, J. Aizpurua, and A. G. Borisov, Faraday Discuss. 214, 147 (2019).

[68] G. Herink, D. R. Solli, M. Gulde, and C. Ropers, Nature (London) 483, 190 (2012).

[69] P. Dombi, A. Hörl, P. Rácz, I. Márton, A. Trügler, J. R. Krenn, and U. Hohenester, Nano Lett. 13, 674 (2013).

[70] C. Li, X. Zhou, F. Zhai, Z. Li, F. Yao, R. Qiao, K. Chen, D. Yu, Z. Sun, K. Liu, and Q. Dai, Appl. Phys. Lett. 111, 133101 (2017).

[71] See Supplemental Material at http://link.aps.org/supplemental/ 10.1103/PhysRevB.101.241412 for the details (i) on the nanoantenna circuit fabrication, (ii) on the TDDFT method, (iii) on the calculation of the electromagnetic field in the gap of the bowtie antenna, and (iv) on the classical simple man's model (SMM) approach. As well, we present an additional analysis of the charge transport and currents in the gap calculated with TDDFT and SMM.

[72] P. B. Corkum, Phys. Rev. Lett. 71, 1994 (1993).

[73] G. G. Paulus, W. Becker, W. Nicklich, and H. Walther, J. Phys. B: At., Mol., Opt. Phys. 27, L703 (1994).

[74] P. D. Keathley, A. Sell, W. P. Putnam, S. Guerrera, L. Velásquez-García, and F. X. Kärtner, Ann. Phys. (NY) 525, 144 (2013).

[75] M. Krüger, M. Schenk, M. Förster, and P. Hommelhoff, J. Phys. B: At., Mol., Opt. Phys. 45, 074006 (2012).

[76] J. W. Gadzuk and E. W. Plummer, Rev. Mod. Phys. 45, 487 (1973).

[77] R. G. Forbes, Appl. Phys. Lett. 89, 113122 (2006).

[78] Note that the field enhancement obtained using the calibration procedure is about seven times smaller than that obtained from classical Maxwell equations. The exact reason for this inconsistency is still under study; however, the results presented below show that the classical time dependence of the field in the gap associated with the field amplitude obtained from the calibration allows one to describe an ensemble of the experimental findings.

[79] This value is obtained considering $E_{\mathrm{gm}}=10 \mathrm{~V} / \mathrm{nm}$ and $1250 \mathrm{~nm}$ carrier wavelength of the single-cycle pulse. 